\title{
Dosimetric comparative study of 3DCRT, IMRT, VMAT, Ecomp, and Hybrid techniques for breast radiation therapy
}

\author{
Semaya Natalia Chen ${ }^{1,2}$, Prabhakar Ramachandran ${ }^{1,3}$, Pradip Deb ${ }^{1}$ \\ ${ }^{1}$ School of Health and Biomedical Sciences, RMIT University, Melbourne, Australia \\ ${ }^{2}$ Department of Radiation Oncology, National Cancer Centre Singapore, Singapore \\ ${ }^{3}$ Princess Alexandra Hospital, Queensland, Australia
}

Received: August 28, 2020

Revised: October 21, 2020

Accepted: November 9, 2020

Correspondence:

Pradip Deb

Medical Radiations, School of Health and Biomedical Sciences, RMIT

University, Victoria, Australia

Tel: +61 399257324

E-mail:Pradip.Deb@rmit.edu.au

ORCID:

https://orcid.org/0000-0003-3612-0859
Purpose: To assess and compare the dosimetric parameters obtained between three-dimensional conformal radiotherapy (3DCRT), three-dimensional field-in-field (3DFIF), 5-field intensity-modulated radiotherapy (IMRT MF5), tangential IMRT (tIMRT), tangential volumetric modulated arc therapy (tVMAT), electronic tissue compensation (Ecomp), and Hybrid treatment plans.

Material and Methods: Thirty planning computed tomography datasets obtained from patients previously treated with whole breast radiation therapy (WBRT) were utilized in this study. Treatment plans were created for 3DCRT, 3DFIF, IMRT MF5, tIMRT, tVMAT, Ecomp, and Hybrid techniques using Eclipse Treatment Planning System (version 13.6) with a prescribed dose of $42.5 \mathrm{~Gy}$ in 16 fractions. Results: Techniques with tangential beams produced statistically significantly better organs-at-risk (OARs) dosimetry $(p<0.001)$. Planning target volume Homogeneity Index $(\mathrm{HI})$ was found to be significantly different among all techniques ( $p<0.001)$, with Ecomp resulting in better HI $(1.061 \pm$ 0.029). Ecomp was also observed to require relatively shorter planning time $(p<0.001)$.

Conclusions: Techniques using tangential fields arrangements produced improved OARs dosimetry. Of all the treatment planning techniques employed in this study, Ecomp was found to be relatively easy to plan and produce acceptable dosimetry for WBRT in a short time.

Keywords: Whole breast radiation therapy, Conformal radiotherapy, Intensity modulated radiotherapy, Electronic tissue compensation

\section{Introduction}

Breast cancer refers to cancer originating in the breast tissues, including the ducts and lobules [1]. According to the latest statistics published by the International Agency for Research on Cancer (IARC), breast cancer ranks as the second most commonly diagnosed cancer in the world. It also highlighted breast cancer as the most common cancer in females worldwide and is the first and second leading cause of all cancer-associated deaths in females in developing and developed regions, respectively [2]. Implementation of breast screening programmes have brought forward the lead time for detecting early breast cancer enabling improved treatment outcome [3]. For patients diagnosed with early-stage breast cancer, conservative management approach of breast-conserving surgery (BCS) is preferred over radical mastectomy commonly used in patients with late-stage breast cancer. The 13th St. Gallen International Breast Cancer Conference (2013) Expert Panel [4] however highlighted that unless post-operative radiation therapy could be delivered, BCS should be reconsidered. Addition of radiation therapy would help to reduce recurrence rates by eradicating the likely presence of microscopic disease after surgery [5]. Currently, radiation therapy presents as an integral component of early-stage, localised breast cancer treatment [5,6]. A large meta-analysis [7] found that radiation therapy delivered post-surgery resulted in ap-

Copyright (C) 2020 The Korean Society for Radiation Oncology

This is an Open Access article distributed under the terms of the Creative Commons Attribution Non-Commercial License (http://creativecommons.org/licenses/by-nc/4.0/) which permits unrestricted non-commercial use, distribution, and reproduction in any medium, provided the original work is properly cited. 
parent reduction in the risk of local recurrences and mortality rate. In addition to improving the local and regional control, use of radiation therapy post-surgery for early-stage breast cancer treatment, also provided the advantage of maintaining patients' quality of life (QOL) to a certain extent. This was attributed to the ability of keeping their breasts, thus maintaining their body image and femininity $[6,8]$.

Radiation therapy is an integral component of early-stage breast cancer management and typically applied to the whole breast post-BCS [9]. Results obtained from randomized trials by the Early Breast Cancer Trialists' Collaborative Group (EBCTCG) [7] revealed that whole breast radiation therapy (WBRT) post-BCS reduced rate of disease recurrence by half and mortality rate by a 6th for patients with early-stage breast cancer. Conventional approach of WBRT is with the use of three-dimensional conformal radiation therapy (3DCRT). However, this approach is associated with the challenge of achieving uniform target dose distribution [10] and introduction of undesired dose to the skin and adjacent organs, resulting in acute and long-term toxicities [6]. These issues stemmed from the steep breast contour change in the 3D shape of the breast and the proximity of target volume to critical organs and tissues. To address these challenges, advanced and complex radiation therapy technology have been developed over the years to increase target dose uniformity, reduce high-dose regions and doses to organs-at-risk (OARs). These are manifested in techniques such as intensity-modulated radiation therapy (IMRT), volumetric modulated arc therapy (VMAT), and electronic tissue compensation (Ecomp). However, there are trade-offs involved in these techniques in an attempt to improve the planning target volume (PTV) and OARs dosimetry. It was also documented in literature that several institutions and radiation therapy departments utilize varying treatment techniques and fractionation schedules for breast radiation treatments $[11,12]$. These differences could result in overall compromised quality of patient care, manpower, available resources and productivity of the department. Additionally, consensus on the optimal technique to be employed for WBRT is still lacking both in literature and clinically. There is also currently limited clinical implementation, research and data available on the use of other advanced techniques like Ecomp and Hybrid for WBRT compared to the other more commonly utilized techniques. Hence, the full extent of their effectiveness on breast cancer treatment remains only partially understood. Therefore, this study aims to: (1) assess the PTV dosimetry and determine the doses delivered to OARs for WBRT using the various treatment planning techniques; (2) evaluate and compare all the dosimetric parameters obtained for both PTV and OARs between the various techniques; and (3) identify an optimal tech- nique that would be recommended for WBRT based on the comparison results.

\section{Materials and Methods}

\section{Patient selection}

This retrospective study utilized thirty anonymized planning computed tomography (CT) datasets of early-stage female breast cancer patients previously treated with WBRT. To maintain diversity of breast sizes and shapes, 13 right-sided and 17 left-sided cases with various breast volumes and separations were selected (Table 1). Every identifiable patient data was replaced with unique numbers as part of de-identification step, according to the centre's ethics protocol.

Planning CT datasets were acquired using Philips CT Big Bore (Philips Healthcare, Best, The Netherlands) with patients lying supine on a lift-up board and arms raised above the head. Each slice of acquired CT datasets was $3 \mathrm{~mm}$ in thickness. Image registration and delineation of gross tumour volume (GTV), PTV and OARs were performed using Eclipse Treatment Planning System (TPS) (version 13.6.23; Varian Medical Systems, Palo Alto, CA, USA). GTV and PTV were contoured by the radiation oncologist. Contoured OARs included contralateral breast (CB), heart, liver, left lung, right lung, and total bilateral lungs. Lung volumes were contoured using auto-threshold function of the planning system. Heart volume was contoured based the heart atlas guidelines. Both CB and liver were delineated based on the visible breast and liver tissues, respectively.

\section{Treatment planning}

As conventional 3DCRT and incorporation of field-in-fields (3DFIF) is still considered to be the standard of care in breast cancer radiation treatment in many institutions, they were also included in this study for dosimetric comparison. For each dataset, 7 distinct plans, namely conventional 3DCRT, 3DFIF, Ecomp, Hybrid, tIMRT, IMRT MF5, and tVMAT were generated and compared against each other. In the case of Hybrid, tIMRT, IMRT MF5, and tVMAT treatment planning techniques, a separate structure encompassing the entire affected breast was contoured and labelled as IMRT PTV for each dataset. All treatment plans were produced in Eclipse TPS at RMIT University. A reference point at isocentre location was used to nor-

Table 1. Range of breast volumes and breast separations $(n=30)$

\begin{tabular}{lcc}
\hline & Mean \pm SD & Median (range) \\
\hline Breast volume $\left(\mathrm{cm}^{3}\right)$ & $1,127.28 \pm 559.54$ & $1,089.78(433.21-2,573.83)$ \\
Breast separation $(\mathrm{cm})$ & $21.70 \pm 2.72$ & $21.76(17.08-27.36)$ \\
\hline
\end{tabular}

$\mathrm{SD}$, standard deviation. 
Table 2. Planning goals for PTV, IL, CL, CB, heart, and liver

\begin{tabular}{|c|c|}
\hline Structure & Planning goals \\
\hline \multirow[t]{2}{*}{ PTV } & Minimum dose $\left(\mathrm{PTV}_{\text {Dmin }}\right)=95 \%$ dose $(40.38 \mathrm{~Gy})$ \\
\hline & Maximum dose $\left(\mathrm{PTV}_{\text {Dmax }}\right)=107 \%$ dose (45.48 Gy) \\
\hline \multirow[t]{3}{*}{ IL } & Percentage volume of IL receiving 5 Gy $\left(\mathrm{IL}_{V_{5 G}}\right)<60 \%$ \\
\hline & Percentage volume of IL receiving $20 \mathrm{~Gy}\left(\mathrm{IL}_{\sqrt{20 \mathrm{G}}}\right)<30 \%$ \\
\hline & Percentage volume of IL receiving $30 \mathrm{~Gy}\left(\mathrm{IL}_{\mathrm{L}_{30 \mathrm{G}}}\right)<10 \%$ \\
\hline $\mathrm{CL}$ & Percentage volume of $\mathrm{CL}$ receiving $10 \mathrm{~Gy}\left(\mathrm{CL}_{\mathrm{V} 10 \mathrm{G} y}\right)=0 \%$ \\
\hline \multirow[t]{2}{*}{$\mathrm{CB}$} & Mean $\mathrm{CB}$ dose $\left(\mathrm{CB}_{\text {Dmean }}\right)<2.5 \mathrm{~Gy}$ \\
\hline & Percentage volume of $\mathrm{CB}$ receiving $5 \mathrm{~Gy}\left(\mathrm{CB}_{\sqrt{56 \mathrm{y}}}\right)<15 \%$ \\
\hline \multirow[t]{3}{*}{ Heart } & Mean heart dose $\left(\right.$ Heart $\left._{\text {Dmean }}\right)<4 \mathrm{~Gy}$ \\
\hline & Percentage volume of heart receiving 5 Gy $\left(\right.$ Heart $\left._{V 5 G y}\right)<40 \%$ \\
\hline & Percentage volume of heart receiving $20 \mathrm{~Gy}\left(\right.$ Heart $\left._{\mathrm{V} 20 \mathrm{~Gy}}\right)<10 \%$ \\
\hline Liver & Mean liver dose $\left(\right.$ Liver $\left._{\text {Dmean }}\right)<20$ Gy \\
\hline
\end{tabular}

PTV, planning target volume; IL, ipsilateral lung; $\mathrm{CL}$, contralateral lung; $\mathrm{CB}$, contralateral breast.

malize the dose. A prescription dose of $42.5 \mathrm{~Gy}$ in 16 fractions was applied for all planning techniques. Each treatment plan was created to meet the planning goals defined in Table 2 .

\section{1) 3DCRT and 3DFIF planning}

3DCRT plans involved a pair of tangential fields with parallel opposing posterior fields and the choices of gantry angles for 3DCRT plans were selected to provide best PTV dose coverage while minimizing as much exposure as possible to adjacent OARs. Dynamic wedge angles and weightings used were selected to give the best PTV dose coverage and homogeneity. For all 3DCRT plans, 6-MV beams were used. Corresponding 3DFIF plan was created using the same gantry and collimator angles as 3DCRT plan for each patient CT dataset. One to two subfields were incorporated and shaped using multi-leaf collimators (MLCs) to remove any hot spots. For breast separation $>23 \mathrm{~cm}, 18-\mathrm{MV}$ beams were included as subfield(s) to produce increased PTV dose coverage.

\section{2) Ecomp planning}

Ecomp plans were generated with the same tangential fields' arrangement used in both 3DCRT and 3DFIF plans for each patient CT dataset. Addition of fluence was performed by incorporating irregular surface compensator for each tangential field. Selection of tissue penetration depth (TPD) for each irregular surface compensator was dependent on each patient's breast separation. Skin flash was added for both fields to provide additional fluence beyond the skin surface. If required, manual fluence editing was also performed on case by case.

\section{3) Hybrid planning}

Hybrid plan consisted of a pair of open tangents supplemented

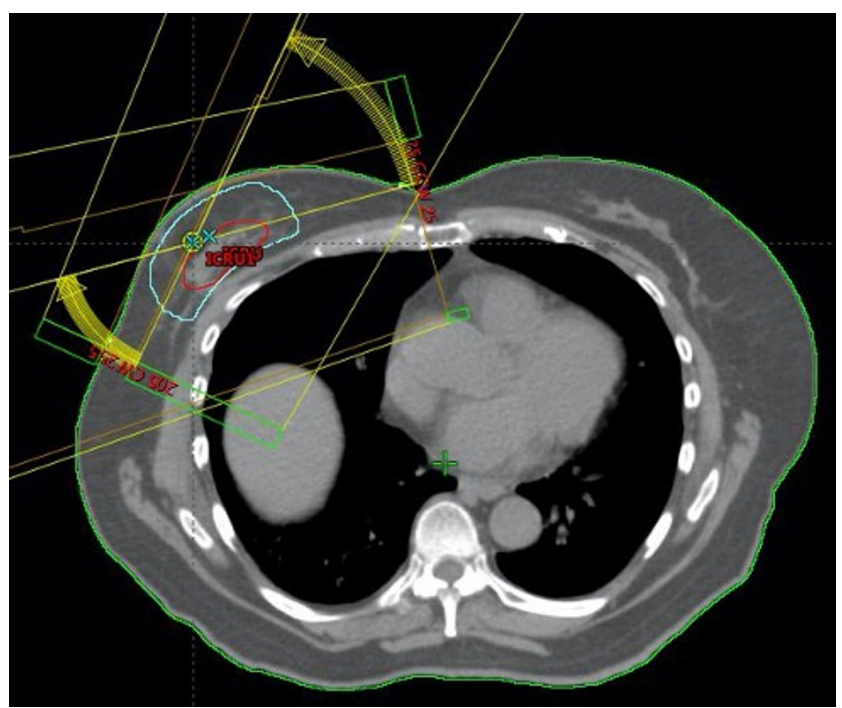

Fig. 1. Tangential $50^{\circ}$ arcs in tangential volumetric modulated arc therapy plan. Each arc travels in opposing direction to each other (clockwise and anti-clockwise) to deliver dose to the entire breast volume.

with a pair of IMRT tangents with the same field parameters. Plan was normalized to $100 \%$ at reference point and manual fluence editing done as necessary.

\section{4) tIMRT and IMRT MF5 planning}

Gantry angles and field parameters for tIMRT plans were similar to Ecomp and the objective functions were specified accordingly to achieve the planning goals. For IMRT MF5 plans, 3 additional fields were incorporated in addition to the medial and lateral tangential fields, resulting in a total of 5 fields. Gantry angles were individually selected for each patient CT dataset to achieve optimal target coverage and minimize entry and exit dose to ipsilateral lung (IL), contralateral lung $(\mathrm{CL}), \mathrm{CB}$, and heart. Similar to tIMRT plan, IMRT MF5 plan was also optimized with objective functions specified to achieve the best attainable plan. Post-optimization improvements such as removal of hot spots, increasing PTV coverage and minimizing OARs dose were performed through manual fluence editing.

\section{5) tVMAT planning}

tVMAT plan utilized tangential with $50^{\circ}$ dual arcs (Fig. 1). The gantry angles were chosen to achieve optimal PTV coverage and minimum OAR doses. Objective functions were specified accordingly to achieve plan objectives and dose constraints as illustrated in Table 2.

\section{Plan comparison and statistical analysis}

The parameters used for comparison of all plans were as shown in Table 3. Conformity Index ( $\mathrm{Cl})$ and Homogeneity Index (HI) were 
Table 3. Plan evaluation parameters for PTV, IL, CL, CB, heart, liver, MUs and the body

\begin{tabular}{|c|c|}
\hline & Plan evaluation parameters \\
\hline \multirow[t]{4}{*}{ PTV } & Conformity Index (Cl) \\
\hline & Homogeneity Index (HI) \\
\hline & Maximum PTV dose (PTV Dmax $\left._{\text {max }}\right)$ \\
\hline & Percentage volume of PTV receiving 95\% dose $\left(\mathrm{PTV}_{\mathrm{V95} \%}\right)$ \\
\hline \multirow[t]{4}{*}{ IL } & Mean IL dose $\left(\mathrm{IL}_{\text {Dmean }}\right)$ \\
\hline & Percentage volume of IL receiving 5 Gy $\left(\mathrm{IL}_{\sqrt{5 G}}\right)$ \\
\hline & Percentage volume of IL receiving $20 \mathrm{~Gy}\left(\mathrm{IL}_{\mathrm{V}_{20 G \mathrm{G}}}\right)$ \\
\hline & Percentage volume of IL receiving $30 \mathrm{~Gy}\left(\mathrm{IL}_{\mathrm{V}_{30 G y}}\right)$ \\
\hline \multirow[t]{2}{*}{$\mathrm{CL}$} & Mean $\mathrm{CL}$ dose $\left(\mathrm{CL}_{\text {Dmean }}\right)$ \\
\hline & Percentage volume of $\mathrm{CL}$ receiving $10 \mathrm{~Gy}\left(\mathrm{CL}_{\mathrm{V}_{10 G y}}\right)$ \\
\hline \multirow[t]{3}{*}{$\mathrm{CB}$} & Maximum $\mathrm{CB}$ dose $\left(\mathrm{CB}_{\mathrm{Dmax}}\right)$ \\
\hline & Mean $\mathrm{CB}$ dose $\left(\mathrm{CB}_{\text {Dmean }}\right)$ \\
\hline & Percentage volume of $\mathrm{CB}$ receiving $5 \mathrm{~Gy}\left(\mathrm{CB}_{\mathrm{v} 5 \mathrm{G}}\right)$ \\
\hline \multirow[t]{3}{*}{ Heart } & Mean heart dose $\left(\right.$ Heart $\left._{\text {Dmean }}\right)$ \\
\hline & Percentage volume of heart receiving 5 Gy $\left(\right.$ Heart $\left._{v_{5 G y}}\right)$ \\
\hline & Percentage volume of heart receiving $20 \mathrm{~Gy}\left(\right.$ Heart $\left._{\mathrm{V} 20 \mathrm{G} y}\right)$ \\
\hline \multirow[t]{2}{*}{ Liver } & Mean liver dose $\left(\right.$ Liver $\left._{\text {Dmean }}\right)$ \\
\hline & Percentage volume of liver receiving $10 \mathrm{~Gy}\left(\right.$ Liver $\left._{\mathrm{V}_{10 \mathrm{G}}}\right)$ \\
\hline MUs & Total MUs \\
\hline Body & Percentage volume of body receiving $10 \mathrm{~Gy}\left(\right.$ Body $\left._{\mathrm{V}_{10 \mathrm{G}}}\right)$ \\
\hline
\end{tabular}

PTV, planning target volume; IL, ipsilateral lung; $C L$, contralateral lung; $\mathrm{CB}$, contralateral breast; MUs, monitor units.

calculated using the following formulas:

$$
\begin{gathered}
C I=\frac{V_{R I}}{T V} \\
H I=\frac{D_{5}}{D_{95}}
\end{gathered}
$$

$V_{R I}$ represents PTV volume in $\mathrm{cm}^{3}$ receiving 41.38 Gy or $95 \%$ of prescribed dose and TV represents PTV volume in $\mathrm{cm}^{3} . D_{5}$ is minimum dose delivered to $5 \%$ of PTV and $D_{95}$ is the minimum dose of 95\% of PTV.

IBM SPSS Statistics version 25 (IBM Corporation, Armonk, NY, USA) was used for the statistical analysis to compare dosimetric parameters. One-way analysis of variance (ANOVA) tests were done to evaluate the dosimetric differences between all 7 techniques and post-hoc analysis was performed using Tukey's honestly significant difference (HSD) test. Interactions between the planning techniques and breast volumes $\left(<1,000 \mathrm{~cm}^{3}\right.$ and $\left.>1,000 \mathrm{~cm}^{3}\right)$ were also evaluated using two-way ANOVA and Tukey's HSD test for post-hoc analysis. Differences were considered as statistically significant when $p$-value is $<0.05$.

\section{Results}

A total of 30 patients were included in this study, all diagnosed with early-stage malignant neoplasm of the breast prior to treatment. Age range of patients was between 34 and 78 years old. Outcomes for the PTV and OARs dosimetric parameters are shown in Table 4.

\section{PTV dosimetry}

There was a significant difference $(p<0.001)$ in PTV $V_{\text {max }}$ amongst

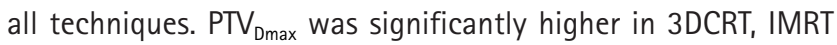
MF5, and tVMAT ( $p<0.001$ ), and above the limit of 107\%. The difference among all 7 techniques in terms of PTV $\mathrm{V}_{\text {v95\% }}$ was statistically significant $(p<0.05)$. Significantly lower PTV v95\% was observed in tVMAT $(p<0.05)$ compared to other 6 techniques. $\mathrm{Cl}$ was found to be significantly different ( $<0.05$ ) among all techniques. tVMAT resulted in the significantly lower $\mathrm{Cl}(\mathrm{p}<0.05)$ compared to all other techniques.

A significant difference $(\mathrm{p}<0.001)$ was observed in terms of $\mathrm{HI}$ amongst all 7 techniques. Statistically significantly higher $\mathrm{HI}$, hence poorer PTV dose homogeneity in 3DCRT, IMRT MF5, and tVMAT ( $p<$ 0.001) were observed when compared to the other techniques. Ecomp was also found to have the best PTV dose homogeneity as demonstrated by the $\mathrm{HI}$ value being closest to 1.0.

Breast volumes of each patient CT dataset was categorised into two groups: $<1,000 \mathrm{~cm}^{3}$ and $>1,000 \mathrm{~cm}^{3}$ to identify dosimetric differences on PTV dose conformity based on $\mathrm{Cl}$ and dose homogeneity based on $\mathrm{HI}$, as a result of interactions between the various techniques and breast volumes. It was shown that there was no statistically significant interaction between the effects of techniques and breast volumes on PTV dose conformity ( $p=0.859$ ) (Table 5, Fig. 2). Like PTV dose conformity, no significant interaction between the effects of techniques and breast volumes was identified on PTV dose homogeneity ( $p=0.550$ ) (Table 5, Fig. 3).

\section{Ipsilateral lung dosimetry}

Difference in $\mathrm{IL}_{\text {Dmean }}$ between the 7 techniques was found to be significant ( $p<0.001)$. tVMAT had significantly highest $\mathrm{IL}_{\text {Dmean }}$ followed by IMRT MF5 ( $p<0.001$ for both) compared to other techniques. No significant difference was observed among techniques with tangential beams.

For $\mathrm{IL}_{\mathrm{V} 5 \mathrm{~Gy}}$ it was also found to have significant difference among all techniques ( $p<0.001$ ). I $\mathrm{L}_{\sqrt{56 \mathrm{G}}}$ showed significantly highest for IMRT MF5 followed by tVMAT ( $p<0.001$ for both). Between IMRT MF5 and tVMAT, IMRT MF5 resulted in significantly higher I $\mathrm{L}_{\sqrt{56 y}}(\mathrm{p}$ $<0.001)$.

Difference in $\mathrm{I}_{\mathrm{V}_{20 G y}}$ among all techniques was statistically signif- 
Table 4. Dosimetric parameter outcomes for PTV and OARs, along with required planning times required between the various techniques

\begin{tabular}{|c|c|c|c|c|c|c|c|}
\hline Parameter & 3DCRT & 3DFIF & IMRT MF5 & tIMRT & tVMAT & Ecomp & Hybrid \\
\hline $\mathrm{PTV}_{\text {Dmax }}$ (Gy) & $46.01 \pm 1.52$ & $45.07 \pm 0.36$ & $46.38 \pm 0.53$ & $45.03 \pm 0.69$ & $46.35 \pm 0.70$ & $44.61 \pm 0.71$ & $44.87 \pm 0.59$ \\
\hline PTV $_{\text {v95\% }}(\%)$ & $98.19 \pm 2.80$ & $97.97 \pm 3.02$ & $99.05 \pm 1.32$ & $98.79 \pm 2.01$ & $95.73 \pm 4.24$ & $98.24 \pm 2.69$ & $98.59 \pm 2.38$ \\
\hline Conformity Index & $0.98 \pm 0.03$ & $0.98 \pm 0.03$ & $0.99 \pm 0.01$ & $0.99 \pm 0.02$ & $0.96 \pm 0.04$ & $0.98 \pm 0.03$ & $0.98 \pm 0.03$ \\
\hline Homogeneity Index & $1.09 \pm 0.03$ & $1.08 \pm 0.03$ & $1.10 \pm 0.01$ & $1.06 \pm 0.03$ & $1.11 \pm 0.03$ & $1.06 \pm 0.03$ & $1.07 \pm 0.03$ \\
\hline $\mathrm{IL}_{\text {Dmean }}(\mathrm{Gy})$ & $4.81 \pm 2.48$ & $4.70 \pm 2.37$ & $8.21 \pm 1.01$ & $4.86 \pm 1.96$ & $8.97 \pm 2.24$ & $5.18 \pm 2.56$ & $5.36 \pm 2.38$ \\
\hline $\mathrm{IL}_{\mathrm{V} 5 \mathrm{y}}(\%)$ & $15.44 \pm 7.84$ & $15.61 \pm 7.88$ & $57.62 \pm 9.20$ & $16.84 \pm 7.34$ & $43.51 \pm 9.87$ & $17.50 \pm 8.74$ & $18.07 \pm 8.35$ \\
\hline $\mathrm{IL}_{\mathrm{V} 20 \mathrm{~Gy}}(\%)$ & $8.61 \pm 5.69$ & $8.58 \pm 5.64$ & $9.13 \pm 3.79$ & $9.23 \pm 5.24$ & $16.68 \pm 5.64$ & $9.94 \pm 6.57$ & $10.44 \pm 6.51$ \\
\hline $\mathrm{IL}_{\sqrt{306 y}}(\%)$ & $7.18 \pm 5.24$ & $7.06 \pm 5.13$ & $2.77 \pm 2.09$ & $6.44 \pm 3.88$ & $9.65 \pm 4.50$ & $7.37 \pm 5.83$ & $7.92 \pm 5.05$ \\
\hline $\mathrm{CL}_{\text {Dmean }}(\mathrm{Gy})$ & $0.04 \pm 0.03$ & $0.04 \pm 0.03$ & $4.00 \pm 1.56$ & $0.04 \pm 0.03$ & $0.47 \pm 0.32$ & $0.05 \pm 0.03$ & $0.06 \pm 0.03$ \\
\hline $\mathrm{CL}_{\mathrm{V} 10 \mathrm{~Gy}}(\%)$ & $0 \pm 0$ & $0 \pm 0$ & $10.38 \pm 6.84$ & $0 \pm 0$ & $0.23 \pm 0.55$ & $0 \pm 0$ & $0 \pm 0$ \\
\hline $\mathrm{CB}_{\mathrm{Dmax}_{\max }}(\mathrm{Gy})$ & $8.02 \pm 12.74$ & $7.87 \pm 12.03$ & $22.00 \pm 11.46$ & $7.25 \pm 11.47$ & $27.61 \pm 7.39$ & $8.20 \pm 12.64$ & $9.29 \pm 13.34$ \\
\hline $\mathrm{CB}_{\text {Dmean }}(\mathrm{Gy})$ & $0.16 \pm 0.12$ & $0.17 \pm 0.12$ & $2.49 \pm 1.83$ & $0.14 \pm 0.13$ & $2.30 \pm 2.08$ & $0.18 \pm 0.15$ & $0.30 \pm 0.53$ \\
\hline $\mathrm{CB}_{\mathrm{V} 5 \mathrm{~Gy}}(\%)$ & $0.12 \pm 0.39$ & $0.16 \pm 0.53$ & $14.52 \pm 17.11$ & $0.13 \pm 0.43$ & $14.86 \pm 15.68$ & $0.17 \pm 0.50$ & $0.18 \pm 0.51$ \\
\hline Heart $_{\text {Dmean }}(G y)$ & $1.52 \pm 1.14$ & $1.51 \pm 1.11$ & $4.20 \pm 0.66$ & $1.80 \pm 0.74$ & $4.98 \pm 1.63$ & $1.91 \pm 0.96$ & $2.15 \pm 1.03$ \\
\hline Heart $_{\mathrm{V} 5 \mathrm{y}}(\%)$ & $2.97 \pm 3.48$ & $2.79 \pm 3.11$ & $23.90 \pm 6.62$ & $4.28 \pm 3.03$ & $25.28 \pm 8.24$ & $4.63 \pm 3.76$ & $5.00 \pm 3.96$ \\
\hline Heart $_{\mathrm{V} 20 \mathrm{~Gy}}(\%)$ & $1.45 \pm 2.26$ & $1.45 \pm 2.26$ & $1.12 \pm 2.07$ & $1.99 \pm 1.95$ & $6.80 \pm 4.19$ & $2.58 \pm 2.52$ & $2.89 \pm 2.77$ \\
\hline Liver $_{\text {Dmean }}(\mathrm{Gy})$ & $1.22 \pm 1.78$ & $1.20 \pm 1.72$ & $6.56 \pm 4.02$ & $1.48 \pm 1.30$ & $4.23 \pm 3.05$ & $1.69 \pm 1.61$ & $1.82 \pm 1.48$ \\
\hline Liver $_{\text {V10Gy }}(\%)$ & $1.73 \pm 4.19$ & $1.76 \pm 4.21$ & $27.00 \pm 20.02$ & $3.00 \pm 3.54$ & $12.89 \pm 10.27$ & $3.27 \pm 3.91$ & $3.55 \pm 3.94$ \\
\hline Body $_{\mathrm{V}_{10 G \mathrm{y}}}(\%)$ & $8.07 \pm 2.08$ & $8.12 \pm 2.10$ & $16.71 \pm 3.65$ & $8.84 \pm 1.82$ & $13.40 \pm 2.71$ & $9.13 \pm 1.97$ & $9.29 \pm 1.97$ \\
\hline Total MUs & $311 \pm 21$ & $303 \pm 15$ & $142 \pm 19$ & $500 \pm 78$ & $364 \pm 49$ & $520 \pm 42$ & $465 \pm 53$ \\
\hline Planning time (min) & $14 \pm 2$ & $16 \pm 3$ & $80 \pm 14$ & $16 \pm 2$ & $39 \pm 2$ & $15 \pm 3$ & $18 \pm 2$ \\
\hline
\end{tabular}

Values are presented as mean \pm standard deviation.

PTV, planning target volume; OAR, organs-at-risk; 3DCRT, three-dimensional conformal radiation therapy; 3DFIF, three-dimensional field-in-field; IMRT MF5, 5-field intensity-modulated radiotherapy; tIMRT, tangential IMRT; tVMAT, tangential volumetric modulated arc therapy; Ecomp, electronic tissue compensation; IL, ipsilateral lung; $\mathrm{CL}$, contralateral lung; $\mathrm{CB}$, contralateral breast; $\mathrm{MUs}$, monitor units.

Table 5. Comparison of PTV Conformity Index (Cl) and Homogeneity Index (HI)

\begin{tabular}{lccccc}
\hline \multirow{2}{*}{ Technique } & \multicolumn{2}{c}{$\mathrm{Cl}(\mathrm{p}=0.859)$} & & \multicolumn{2}{c}{$\mathrm{HI}(\mathrm{p}=0.550)$} \\
\cline { 2 - 3 } \cline { 5 - 6 } 3DCRT & Breast volume $<1,000 \mathrm{~cm}^{3}$ & Breast volume $>1,000 \mathrm{~cm}^{3}$ & & Breast volume $<1,000 \mathrm{~cm}^{3}$ & Breast volume $>1,000 \mathrm{~cm}^{3}$ \\
3DFIF & $0.97 \pm 0.04$ & $0.99 \pm 0.02$ & $0.98 \pm 0.03$ & $1.09 \pm 0.03$ & $1.09 \pm 0.04$ \\
IMRT MF5 & $0.98 \pm 0.03$ & $0.99 \pm 0.01$ & $0.99 \pm 0.01$ & $1.07 \pm 0.02$ & $1.08 \pm 0.04$ \\
tIMRT & $0.99 \pm 0.02$ & $0.99 \pm 0.02$ & $1.10 \pm 0.01$ & $1.09 \pm 0.01$ \\
tVMAT & $0.95 \pm 0.04$ & $0.96 \pm 0.05$ & $1.06 \pm 0.03$ & $1.07 \pm 0.03$ \\
Ecomp & $0.98 \pm 0.03$ & $0.99 \pm 0.03$ & $1.12 \pm 0.03$ & $1.11 \pm 0.03$ \\
Hybrid & $0.98 \pm 0.02$ & $0.099 \pm 0.03$ & $1.06 \pm 0.03$ & $1.06 \pm 0.03$ \\
\hline
\end{tabular}

Values are presented as mean \pm standard deviation.

PTV, planning target volume; 3DCRT, three-dimensional conformal radiation therapy; 3DFIF, three-dimensional field-in-field; IMRT MF5, 5-field intensity-modulated radiotherapy; tIMRT, tangential IMRT; tVMAT, tangential volumetric modulated arc therapy; Ecomp, electronic tissue compensation.

icant $(p<0.001)$. Statistically significantly higher $\mathrm{IL}_{\mathrm{V}_{20 G}}$ was found in tVMAT ( $p<0.001$ ) in comparison to other techniques. The difference among all techniques was statistically significant for $\mathrm{IL}_{\mathrm{V}_{30 G y}}$ ( $p<0.001)$. IMRT MF5 showed significantly reduced $\mathrm{IL}_{\mathrm{V}_{306}}(\mathrm{p}<$ $0.05)$ in comparison to other techniques.

\section{Contralateral lung dosimetry}

The difference among all techniques in terms of $\mathrm{CL}_{\text {Dmean }}$ was statis- tically significant ( $p<0.001$ ). IMRT MF5 was found to result in statistically significantly higher $\mathrm{CL}_{\text {Dmean }}(\mathrm{p}<0.001)$ compared to other techniques, close to 100 times higher than techniques with tangential fields arrangement. tVMAT followed as the second highest in terms of $\mathrm{CL}_{\text {Dmean }}$ and was approximately 10 times higher than 3DCRT, 3DFIF, tIMRT, Ecomp, and Hybrid. Among techniques using tangential fields arrangement, no significant difference was observed. 


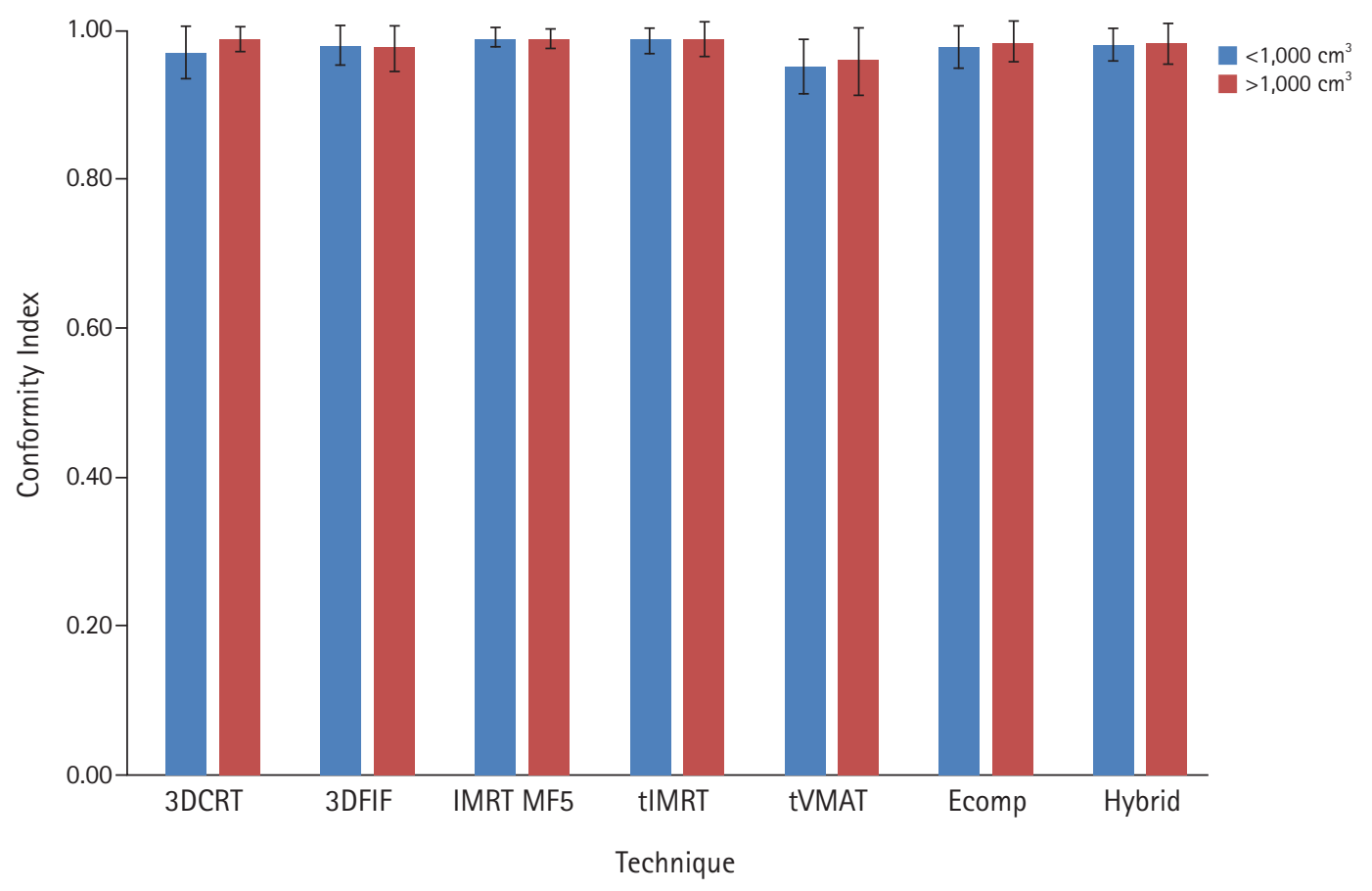

Fig. 2. Mean Conformity Index $(\mathrm{Cl})$ of all 7 techniques according to breast volumes. Error bars represent \pm 1 standard deviation. Black line over bars represents $\mathrm{Cl}$ of 1.0 that represents ideal dose conformity to planning target volume. 3DCRT, three-dimensional conformal radiation therapy; 3DFIF, three-dimensional field-in-field; IMRT MF5, 5-field intensity-modulated radiotherapy; tIMRT, tangential IMRT; tVMAT, tangential volumetric modulated arc therapy; Ecomp, electronic tissue compensation.

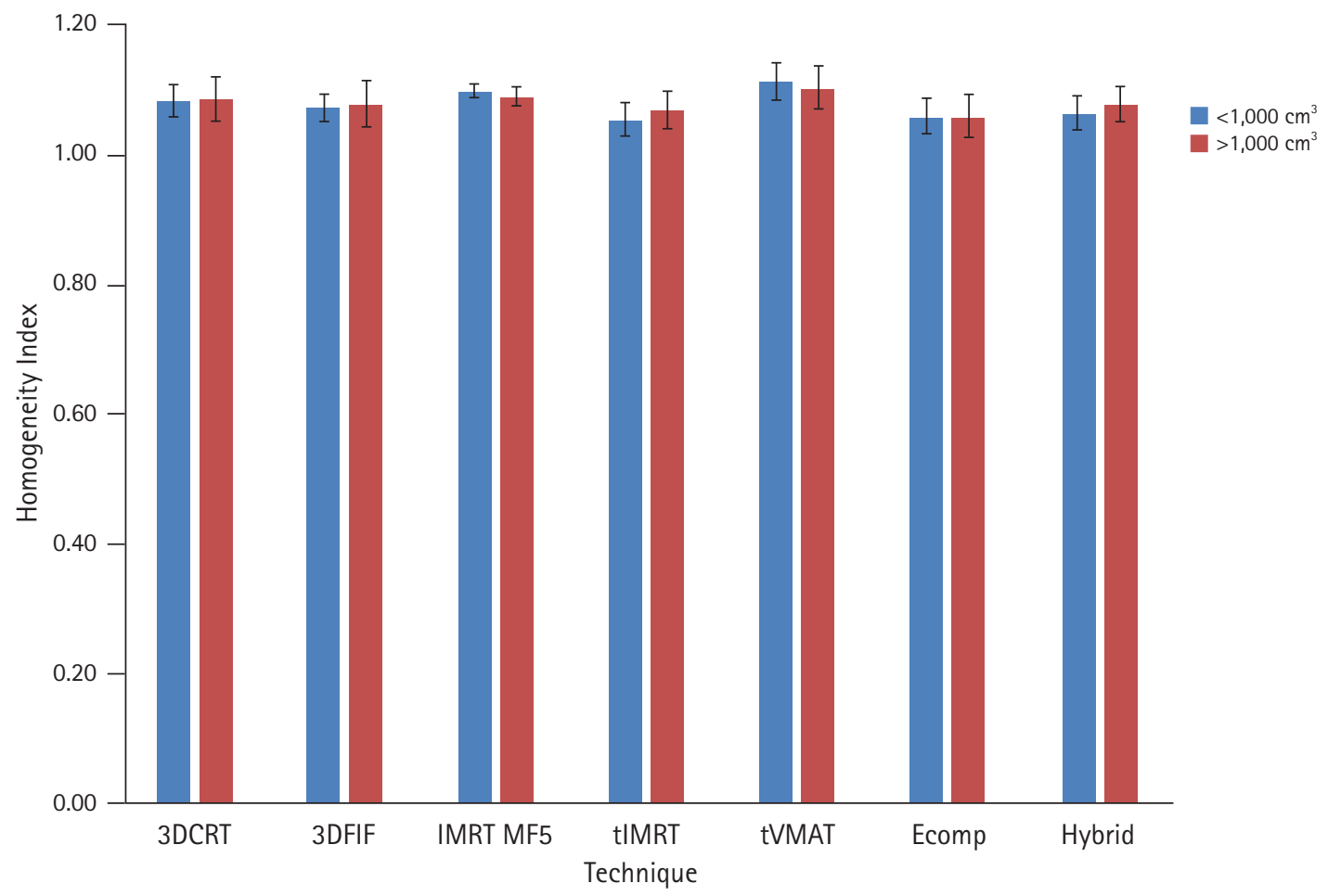

Fig. 3. Mean Homogeneity Index (HI) of all 7 techniques according to breast volumes. Error bars represent \pm 1 standard deviation. Black line over bars represents $\mathrm{HI}$ of 1.0 that represents ideal dose homogeneity in planning target volume. 3DCRT, three-dimensional conformal radiation therapy; 3DFIF, three-dimensional field-in-field; IMRT MF5, 5-field intensity-modulated radiotherapy; tIMRT, tangential IMRT; tVMAT, tangential volumetric modulated arc therapy; Ecomp, electronic tissue compensation. 
Similar to $\mathrm{CL}_{\text {Dmean }}$ difference in $\mathrm{CL}_{\mathrm{V} 10 \mathrm{~Gy}}$ was also statistically significant among all techniques ( $p<0.001$ ). IMRT MF5 produced significantly increased $\mathrm{CL}_{\sqrt{10 G y}}(\mathrm{p}<0.001)$ compared to others.

\section{Contralateral breast dosimetry}

Difference among the 7 techniques was statistically significant ( $p$ $<0.001$ ) for $\mathrm{CB}_{\text {Dmax }}$. Both tVMAT and IMRT MF5 ( $p<0.001$ for both) resulted in statistically significantly increased $\mathrm{CB}_{\text {Dmax }}$. However, both were not significantly different to each other. There was also no statistically significant difference in $\mathrm{CB}_{\text {Dmax }}$ demonstrated between the techniques with tangential beams.

$\mathrm{CB}_{\text {Dmean }}$ of all techniques were significantly different $(\mathrm{p}<0.001)$. $\mathrm{CB}_{\text {Dmean }}$ was significantly higher in IMRT MF5 and tVMAT ( $p<$ 0.001 for both) compared to other techniques, however both were not different from each other.

$\mathrm{CB}_{\mathrm{V} 5 \mathrm{G}}$ of all techniques were also shown to be statistically significantly different ( $p<0.001)$. tVMAT and IMRT MF5 ( $p<0.001$ for both) resulted in almost 100 times significantly higher $\mathrm{CB}_{\mathrm{V} 5 \mathrm{G}}$ compared to other 5 techniques with tangential beams, however, were not significantly different from each other.

\section{Heart dosimetry}

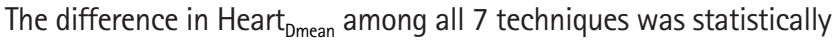
significant ( $p<0.001$ ). Statistically significantly increased Heart ${ }_{D-}$ mean was observed in TVMAT and IMRT MF5 ( $p<0.001$ for both) and were beyond the dose tolerance goal limit of $4 \mathrm{~Gy}$. However, no significant difference was observed between both techniques. Between techniques with tangential fields arrangement, no statistically significant difference was found.

In terms of Heart ${ }_{\mathrm{V} 5 \mathrm{~Gy}}$ the difference between all techniques was also significant $(p<0.001)$. Significantly higher Heart $_{\text {v5Gy }}$ in tVMAT and IMRT MF5 ( $p<0.001$ for both) were observed, approximately 5 to 10 times greater than other 5 techniques.

For Heart ${ }_{\mathrm{V} 20 \mathrm{~Gy}}$ the difference among all techniques was significant ( $p<0.001)$. tVMAT demonstrated significantly highest Heartv20Gy compared to other techniques ( $p<0.001)$.

\section{Liver dosimetry}

IMRT MF5 resulted in significantly higher $\operatorname{Liver}_{\text {Dmean }}(p<0.001)$ compared to 3DCRT, 3DFIF, tIMRT, Ecomp, and Hybrid. tVMAT ( $p<$ 0.05) also had significantly higher Liver $_{\text {Dmean }}$ compared to 3DCRT and 3DFIF, however was not significantly different from tIMRT, Ecomp, Hybrid, and IMRT MF5. The remaining 5 techniques adopting the tangential fields configuration showed similar Liver $_{\text {Dmean }}$ and were not significantly different from each other.

Liver $_{\text {V10Gy }}$ was also significantly different between the various techniques ( $p<0.001$ ). IMRT MF5 demonstrated statistically sig- nificantly highest Liver $_{\text {V10Gy }}$ among all techniques $(p<0.05)$. tVMAT $(p<0.05)$ also had significantly higher Liver $_{\text {v10Gy }}$ compared to 3DCRT and 3DFIF, however was not statistically significantly different from other intensity-modulated techniques-tIMRT, Ecomp, and Hybrid. Reduced Liver ${ }_{\text {V10Gy }}$ was observed among techniques with tangential beams, however no significant difference was observed among them.

\section{Total monitor units, body low dose and planning time comparisons}

Difference in total monitor units (MUs) produced by all 7 techniques was statistically significant $(p<0.001)$. Statistically significantly highest total MUs was observed in IMRT MF5 ( $p<0.001$ ). 3DCRT, 3DFIF, and tVMAT were shown to produce significantly lower total MUs ( $p<0.001$ for all three) but were not significantly different from each other. Those utilizing intensity-modulated beams (tIMRT, Ecomp and Hybrid), resulted in significantly higher total MUs ( $p<0.001$ for all three). However, they were also not significantly different from each other.

Statistically significant increase in Body ${ }_{\mathrm{V}_{10 G}}$ of approximately twice the amount was observed in IMRT MF5 and TVMAT ( $p<$ 0.001 for both) among all techniques.

To evaluate the efficiency of each technique, planning times for the various techniques were also recorded and compared (Table 4). Planning times for all studied techniques were significantly different ( $p<0.001)$. Among all techniques, IMRT MF5 required the longest planning time followed by tVMAT ( $p<0.001$ for both). IMRT MF5 was significantly longer $(p<0.001)$ to plan than tVMAT. 3DCRT and Ecomp were techniques with shortest planning times.

\section{Discussion and Conclusion}

To date, no study had been carried out that performed a direct comparison of conventional 3DCRT and 3DFIF with more advanced techniques: Ecomp, Hybrid technique, various numbers of IMRT beams and use of tangential arc arrangements in VMAT, as part of an entire study of radiation therapy to both left and right early-stage breast cancer cases. In this study, PTV and OARs dosimetries were used as parameters for direct comparison between 3DCRT, 3DFIF, IMRT MF5, tIMRT, tVMAT, Ecomp, and Hybrid techniques.

It is essential that PTV Dmax $_{\text {ma }}$ is kept to $<107 \%$ (45.48 Gy) to achieve the desired dose uniformity eventually to reduce risk of skin reactions. Results obtained in this study demonstrated that only 3DFIF, tIMRT, Ecomp, and Hybrid were able to meet the constraint of PTV Dmax $<107 \%$, with Ecomp having the lowest PTV $\mathrm{Dmax}_{\text {- }}$

IMRT MF5 produced highest PTV $\mathrm{V}_{\text {95\% }}$ compared to other tech- 
niques, indicating improved dose coverage. This was consistent with the findings in some studies that increasing number of IMRT beams would increase PTV dose coverage [13-15]. As PTV Cl is directly correlated to $\mathrm{PTV}_{\mathrm{V} 95 \%}$, it was hence not surprising that IMRT MF5 also similarly showed highest $\mathrm{Cl}$ among all techniques. Despite having the highest $\mathrm{PTV}_{\mathrm{v95 \%}}$ and $\mathrm{Cl}$ value closest to the ideal value of 1.0, IMRT MF5 did not show any significant difference in comparison with techniques using tangential beams. This indicates that although increasing number of beams as seen in IMRT MF5 showed increased $\mathrm{PTV}_{\mathrm{v} 95 \%}$ and $\mathrm{Cl}$, it is however comparable to techniques using only tangential beams.

tVMAT showed the statistically significantly worst performance for $\mathrm{PTV}_{\text {v95\% }}$ and $\mathrm{Cl}$. This was in contrary to the findings reported by Zhao et al. [16], Lin et al. [17], and Qiu et al. [18] that VMAT resulted in improved $\mathrm{Cl}$ than IMRT and 3DCRT plans. This discrepancy can be attributed to the varied arc arrangement used in this study compared to those used by the above-mentioned studies. As a matter of fact, Viren et al. [19] found that use of a pair of $50^{\circ}$ dual arcs, similar to that used in this study, resulted in lower target dose coverage and conformity compared to the use of continuous arc arrangement employed by most studies.

Previous reports stated Ecomp as providing improved target dose homogeneity, especially over the conventional 3DCRT [20-22]. This was observed as true in this study, with Ecomp producing the best $\mathrm{HI}$ among all techniques, having the value closest to the ideal value of 1.0. Zaghloul et al. [23] reported a correlation between improved PTV $\mathrm{HI}$ and significant reduction of acute skin reaction incidence. This correlation between the clinical outcome and PTV HI can be applicable to the findings in this study that indicates Ecomp as having the potential of reducing risk of acute skin toxicities compared to the other techniques. In contrast, tVMAT and IMRT MF5 were observed to give statistically significantly poorest PTV HI. Poorer PTV dose homogeneity observed in both tVMAT and IMRT MF5 could be due to number of beams and the configurations of arcs and beams used. The use of optimizer and OARs constraint-based process involved, could have also further contributed to the overall poorer PTV dose homogeneity. In the attempt of meeting the OARs dose constraint, optimization-convergence errors were likely to have occurred [24], especially due to the major tissue density difference present between the breast tissue and air in the lungs. Thus, in the optimizer's attempt of achieving various constraints of OARs that also included the low-density lungs, heterogenous regions of high and low doses (hot and cold spots) were formed within the PTV.

Previous studies also reported a correlation in breast volume and dose homogeneity [25-27]. Large breast volumes tend to involve reduced dose homogeneity and increased high dose regions within the PTV that result in acute skin toxicity $[15,26]$. In this study, it was found that there was no statistically significant interaction between the effects of variations in breast volumes and techniques on both $\mathrm{Cl}$ and $\mathrm{HI}$. However, there were significant differences on PTV $\mathrm{Cl}$ and $\mathrm{HI}$ between various techniques. This indicates that only variations in techniques affect $\mathrm{Cl}$ and $\mathrm{HI}$, and that they do not vary according to breast volumes.

In this study, IMRT MF5 had the highest I $L_{\sqrt[V]{56}}$ and lowest I $L_{V_{30 G y}}$ among all techniques. These findings corroborate with the data reported by Liu et al. [28] that showed 5-field IMRT was able to reduce the $I_{V_{20 G y}}$ and $I_{L_{30 G y}}$ in comparison to 3DCRT with tangential beams. In terms of CL exposure, IMRT MF5 also showed significantly highest $\mathrm{CL}_{\mathrm{Dmean}}$ and $\mathrm{CL}_{\mathrm{V}_{10 G y}}$. Such findings are similar to that presented by Rongsriyam et al. [29] in which $\mathrm{CL}_{\text {Dmean }}$ was also found to be much higher in IMRT than in 3DCRT. These results were expected due to greater number of beams involved that resulted in

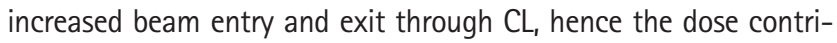
bution. This implies that success of IMRT MF5 in reducing the volumes of lungs receiving high doses comes at the expense of overall higher mean dose and greater volumes receiving the low dose.

Use of arcs in VMAT in previous studies $[14,30]$ was similarly found to result in significantly higher low-dose volume, especially to $\mathrm{IL}$, which is also observed in this present study. In general, this highlights that non-tangential beams techniques result in increased lungs exposure, thus greater implication in future development of pulmonary complications. Techniques with tangential beams are in contrast, comparably similar to each other in improving lung sparing, hence reducing the likelihood of radiation pneumonitis and secondary lung cancer post-radiotherapy.

Some studies have reported that IMRT reduced the CB dose compared to conventional 3DCRT [31,32]. This was found to be true in this study, with tIMRT showing lower CB doses than 3DCRT. However, it is not the case for IMRT MF5 due to greater number of fields used that resulted in low dose spill to greater CB volume. This can be of high importance, especially to patients $<40$ years old who are at greater risk of secondary CB cancer [33]. Similar to the results in this study, other papers have also reported that use of VMAT resulted in high low-dose volume to CB [14,34], hence also posing the issue of greater risk of inducing secondary CB cancers especially to younger patients. The application of techniques with tangential beams in contrast, introduces a greater advantage of improved $\mathrm{CB}$ dosimetry, thus reduced risk of inducing secondary $\mathrm{CB}$ cancers.

In this study, all studied techniques were able to meet the con-

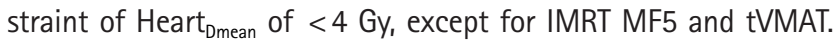
Both the above techniques were also found to result in higher Heart $_{\text {V5Gy }}$ compared to the rest. These findings are similar to those 
by Liu et al. [28] who found that 5-field IMRT and double-arcs VMAT both had higher heart doses compared to 3DCRT. This present study showed reduced heart doses with in techniques utilizing tangential beams, hence once again highlighting the advantage of improved organ sparing compared to the use of multi-fields or arcs utilizing techniques.

Radiation-induced liver disease (RILD) is often described as a significant complication and major limitation of liver cancer radiation treatment [35]. Although the whole liver is not directly irradiated in breast radiation therapy, liver toxicity is a factor that must still be taken into consideration in right-sided breast radiation therapy due to its close proximity to PTV. In this study, the number of right-sided patient cases is less than left-sided cases, however the results positively showed a significant reduction of liver doses in 3DCRT, 3DFIF, tIMRT, Ecomp, and Hybrid compared to IMRT MF5 and tVMAT. Higher liver doses observed in IMRT MF5 and tVMAT can be attributed to greater low dose volume caused by increased number of intensity-modulated fields, greater field scattering and dose leakage between the MLC leaves [28]. Techniques utilizing tangential beams are in contrast better for liver sparing and thus able to reduce the risk of RILD.

IMRT MF5 showed significantly highest total MUs, almost 5 times of the conventional 3DCRT and 3DFIF. Other techniques similarly using intensity-modulated fields (tIMRT, tVMAT, Ecomp, and Hybrid) also showed higher MUs than 3DCRT and 3DFIF. This was expected because intensity-modulated techniques involve large numbers of small subfields to achieve optimum intensity distributions of each treatment field [36], thus resulting in higher MUs compared to non-intensity modulated 3DCRT and 3DFIF. With increase in the number of intensity-modulated beams, it was not surprising that IMRT MF5 resulted in highest total MUs.

In this study, IMRT MF5 had the highest Body ${ }_{\text {viogy }}$ followed by tVMAT. Involvement of more beams and therefore larger volume of normal tissues exposed to the low dose as demonstrated with higher Body ${ }_{\text {V10Gy }}$ was thus expected of IMRT MF5. Although tIMRT, Ecomp, and Hybrid are also intensity-modulated techniques, the lower MUs involved provided the advantage of lower Body v10Gy $_{\text {that }}$ are comparable to conventional 3DCRT, hence pose no increased risk of inducing secondary cancers compared to conventional 3DCRT and 3DFIF.

tVMAT has lower MUs than all other intensity-modulated techniques and is similar to that of 3DCRT and 3DFIF. However, it still resulted in second highest Body ${ }_{\mathrm{V}_{10 G}}$. This finding is attributed to the arc motion that produces dose fall-off occurring in every direction, therefore distributing low doses to bigger volumes of normal tissues, resulting in subsequent increased risk of secondary cancer induction. This observation is similar to a study conducted by
Abo-Madyan et al. [37] who found that in comparison with 3DCRT, VMAT resulted in higher cumulated excess absolute risk of developing secondary malignancies after exposure to low doses. Based on the analysis, use of tIMRT, Ecomp, and Hybrid as alternatives to 3DCRT and 3DFIF pose no increased risk of inducing secondary malignancies due to comparable smaller body volumes receiving low doses. IMRT MF5 and tVMAT however, can potentially increase the risk due to greater body volume exposed to low doses.

A large number of early-stage breast cancer patients is often observed in a typical radiation therapy department. This implies that techniques with increasing complexity will affect the department's resource allocation. To determine the department's resource efficiency, parameters such as treatment and planning times were often used [38]. Under clinical setting, total MUs are associated with treatment time. Though this study is a planning study that does not constitute treatment delivery, the results suggest that IMRT MF5 is the technique that would require longest treatment time while techniques with lowest MUs (3DCRT, 3DFIF and tVMAT) would result in shortest treatment time.

In terms of planning time, this study similarly revealed that IMRT MF5 requires the longest time followed by tVMAT. This was due to the process of adjusting the field and arc arrangements, along with iterative optimization processes to achieve the best plans. Planning times for tIMRT and Ecomp are in contrast, comparable to conventional 3DCRT and 3DFIF. However, as tIMRT requires inverse-planning, similar to IMRT MF5, tVMAT and Hybrid, additional steps of contouring of the IMRT structures are required for the optimization process. The planning times recorded for these inverse-planned techniques in this study do not include the duration required to contour those IMRT structures. Since Ecomp is a forward-planned technique, that additional time of contouring the IMRT structures is not required, hence is more superior than the other advanced techniques in terms of time-efficiency. When looking at studies published on Ecomp, it was also suggested that electronic compensation algorithm used for Ecomp is a feature found only in Eclipse TPS. Hence, institutions using other TPS might not be able to implement the use of Ecomp as an alternative WBRT technique.

Based on the analyses, techniques with tangential beams result in significant reduction of OARs doses compared to those using multiple beams and arcs as demonstrated in IMRT MF5 and tVMAT. In terms of PTV dose coverage, conformity and homogeneity, tIMRT, Ecomp, and Hybrid are more superior than 3DCRT and 3DFIF and are also comparable to conventional 3DCRT and 3DFIF in the OARs doses. However, it is noted that Hybrid led to higher contribution of low-doses to $\mathrm{IL}, \mathrm{CL}, \mathrm{CB}$, heart, and liver compared to tIMRT and Ecomp, indicating a possible clinical significance of increased risk of long-term complications and secondary cancer induction. This 
was similarly observed in the studies conducted by Mayo et al. [39] and Xie et al. [40] who found that improvements of PTV dosimetry in Hybrid were achieved at the expense of increased low-dose volume to the $C B$, lungs and heart compared to tangential IMRT-only technique.

Out of the three, Ecomp required comparably shorter planning time. Use of skin flash tool incorporated in Ecomp also further helps to account for patient's breathing motion, increasing the accuracy of dose delivery. Overall, this highlights the potentially better patient outcome and subsequently improved patient's QOL. Therefore, Ecomp shows promising use in terms of efficiency and effectiveness and may be favoured as an optimal technique for WBRT in departments where Eclipse planning system is available.

This study focusses exclusively on early-stage breast cancer patients treated with WBRT without any regional lymph nodes involvement. Hence, the current results might not be applicable for patients requiring treatments to both breast and the regional nodes. A greater number of patient cases and inclusion of patient groups requiring breast and nodal radiation therapy should therefore be done in future studies to better evaluate the results and draw more definitive conclusions.

In conclusion, techniques with tangential fields arrangement resulted in overall better OARs dosimetry compared to those with multi-fields and arcs arrangements. Of all the techniques used in this study, Ecomp emerged as a better treatment technique for WBRT in terms of both effectiveness and efficiency. However, this study is a retrospective planning study and therefore the planning dosimetric data obtained might be different from actual delivered doses to patients in real-life clinical situations. Further studies involving water phantom simulation and/or clinical applications on patients are hence required to better compare and verify the dosimetric results obtained in this study.

\section{Conflict of Interest}

No potential conflict of interest relevant to this article was reported.

\section{Acknowledgements}

The authors acknowledge the support given by the Medical Radiations Discipline in the School of Health and Biomedical Sciences at RMIT University, Australia.

\section{References}

1. Cancer Australia. What is breast cancer? [Internet]. Strawberry
Hills, Australia: Cancer Australia; c2020 [cited 2020 Nov 15]. Available from: https://breast-cancer.canceraustralia.gov.au.

2. International Agency for Research on Cancer. Breast cancer [Internet]. Lyon, France: International Agency for Research on Cancer; c2020 [cited 2020 Nov 15]. Available from: https://gco.iarc. $\mathrm{fr} /$ today/fact-sheets-cancers.

3. Marmot MG, Altman DG, Cameron DA, Dewar JA, Thompson SG, Wilcox M. The benefits and harms of breast cancer screening: an independent review. Br J Cancer 2013;108:2205-40.

4. Goldhirsch A, Winer EP, Coates AS, et al. Personalizing the treatment of women with early breast cancer: highlights of the St Gallen International Expert Consensus on the Primary Therapy of Early Breast Cancer 2013. Ann Oncol 2013;24:2206-23.

5. Zurrida S, Veronesi U. Milestones in breast cancer treatment. Breast J 2015;21:3-12.

6. Nantavithya C, Saksornchai K, Oonsiri P, Shotelersuk K. Dosimetric study of three-dimensional conformal radiotherapy, electronic compensator technique, intensity-modulated radiation therapy and volumetric-modulated arc therapy in whole breast irradiation. J Radiother Pract 2017;16:431-43.

7. Early Breast Cancer Trialists' Collaborative Group (EBCTCG), Darby $\mathrm{S}, \mathrm{McGale} \mathrm{P}$, et al. Effect of radiotherapy after breast-conserving surgery on 10-year recurrence and 15-year breast cancer death: meta-analysis of individual patient data for 10,801 women in 17 randomised trials. Lancet 2011;378:1707-16.

8. Tenea Cojan TS, Vidrighin CD, Ciobanu M, et al. Breast-conserving surgery in breast cancer. Chirurgia (Bucur) 2012;107:616-25.

9. Moran MS, Schnitt SJ, Giuliano AE, et al. Society of Surgical Oncology-American Society for Radiation Oncology consensus guideline on margins for breast-conserving surgery with wholebreast irradiation in stages I and II invasive breast cancer. Ann Surg Oncol 2014;21:704-16.

10. Zhang $\mathrm{Q}, \mathrm{Yu} \mathrm{XL}, \mathrm{Hu} W \mathrm{WG}$, et al. Dosimetric comparison for volumetric modulated arc therapy and intensity-modulated radiotherapy on the left-sided chest wall and internal mammary nodes irradiation in treating post-mastectomy breast cancer. Radiol Oncol 2015:49:91-8.

11. Dayes IS, Whelan TJ, Julian JA, et al. Cross-border referral for early breast cancer: an analysis of radiation fractionation patterns. Curr Oncol 2006;13:124-9.

12. Kara FG, Haydaroglu A, Eren $H$, Kitapcioglu G. Comparison of different techniques in breast cancer radiotherapy planning. J Breast Health 2014;10:83-7.

13. Popescu CC, Olivotto I, Patenaude V, Wai E, Beckham WA. Inverse-planned, dynamic, multi-beam, intensity-modulated radiation therapy (IMRT): a promising technique when target volume is the left breast and internal mammary lymph nodes. Med Do- 
$\operatorname{sim}$ 2006;31:283-91.

14. Jin GH, Chen LX, Deng XW, Liu XW, Huang Y, Huang XB. A comparative dosimetric study for treating left-sided breast cancer for small breast size using five different radiotherapy techniques: conventional tangential field, filed-in-filed, tangential-IMRT, multi-beam IMRT and VMAT. Radiat Oncol 2013;8:89.

15. Ayata HB, Guden M, Ceylan C, Kucuk N, Engin K. Comparison of dose distributions and organs at risk (OAR) doses in conventional tangential technique (CTT) and IMRT plans with different numbers of beam in left-sided breast cancer. Rep Pract Oncol Radiother 2011;16:95-102.

16. Zhao $H_{1}$ He M, Cheng $G$, et al. A comparative dosimetric study of left sided breast cancer after breast-conserving surgery treated with VMAT and IMRT. Radiat Oncol 2015;10:231.

17. Lin JF, Yeh DC, Yeh HL, Chang CF, Lin JC. Dosimetric comparison of hybrid volumetric-modulated arc therapy, volumetric-modulated arc therapy, and intensity-modulated radiation therapy for left-sided early breast cancer. Med Dosim 2015;40:262-7.

18. Qiu JJ, Chang Z, Wu O, Yoo S, Horton J, Yin FF. Impact of volumetric modulated arc therapy technique on treatment with partial breast irradiation. Int J Radiat Oncol Biol Phys 2010;78:28896.

19. Viren T, Heikkila J, Myllyoja K, Koskela K, Lahtinen T, Seppala J. Tangential volumetric modulated arc therapy technique for left-sided breast cancer radiotherapy. Radiat Oncol 2015;10:79.

20. Alghufaili AH, Shanmugarajah L, Kumaraswamy LK. Correlating the depth of compensation to the 3-D shape of the breast to achieve homogeneous dose distribution using the electronic tissue compensation treatment technique. Med Dosim 2019;44:304.

21. Hideki F, Nao K, Hiroyuki H, Hiroshi K, Haruyuki F. Improvement of dose distribution with irregular surface compensator in whole breast radiotherapy. J Med Phys 2013;38:115-9.

22. Song $Y$, Zhang $M$, Gan $L$, et al. Predictive parameters for selection of electronic tissue compensation radiotherapy in early-stage breast cancer patients after breast-conserving surgery. Oncotarget 2016;7:32835-45.

23. Zaghloul HA, Rashed YA, Shaukat AA, Rostom YA, Badawy SE. Intensity modulated radiotherapy using multiple in fields compared to wedged fields in breast irradiation: clinical impact on early skin reaction and organs at risk doses. J Nucl Med Radiat Ther 2014;5:176.

24. Lu JY, Zhang JY, Li M, et al. A simple optimization approach for improving target dose homogeneity in intensity-modulated radiotherapy for sinonasal cancer. Sci Rep 2015;5:15361.

25. Moody AM, Mayles WP, Bliss JM, et al. The influence of breast size on late radiation effects and association with radiotherapy dose inhomogeneity. Radiother Oncol 1994;33:106-12.

26. De Langhe $S$, Mulliez $T$, Veldeman $L$, et al. Factors modifying the risk for developing acute skin toxicity after whole-breast intensity modulated radiotherapy. BMC Cancer 2014;14:711.

27. Neal AJ, Torr M, Helyer S, Yarnold JR. Correlation of breast dose heterogeneity with breast size using 3D CT planning and dose-volume histograms. Radiother Oncol 1995;34:210-8.

28. Liu H, Chen X, He Z, Li J. Evaluation of 3D-CRT, IMRT and VMAT radiotherapy plans for left breast cancer based on clinical dosimetric study. Comput Med Imaging Graph 2016;54:1-5.

29. Rongsriyam K, Rojpornpradit P, Lertbutsayanukul C, Sanghangthum T, Oonsiri S. Dosimetric study of inverse-planed intensity modulated, forward-planned intensity modulated and conventional tangential techniques in breast consenving radiotherapy. J Med Assoc Thai 2008:91:1571-82.

30. Zheng H, Lai YQ, Zhou Y, et al. A comparative dosimetric study of seven radiation techniques for breast cancer after mastectomy and immediate breast reconstruction. Transl Cancer Res 2017;6: 788-97.

31. Burmeister J, Alvarado N, Way S, et al. Assessment and minimization of contralateral breast dose for conventional and intensity modulated breast radiotherapy. Med Dosim 2008;33:6-13.

32. Bhatnagar AK, Brandner E, Sonnik D, et al. Intensity modulated radiation therapy (IMRT) reduces the dose to the contralateral breast when compared to conventional tangential fields for primary breast irradiation. Breast Cancer Res Treat 2006;96:41-6.

33. Stovall M, Smith SA, Langholz BM, et al. Dose to the contralateral breast from radiotherapy and risk of second primary breast cancer in the WECARE study. Int J Radiat Oncol Biol Phys 2008;72: 1021-30.

34. Osman SO, Hol S, Poortmans PM, Essers M. Volumetric modulated arc therapy and breath-hold in image-guided locoregional left-sided breast irradiation. Radiother Oncol 2014;112:17-22.

35. Kim J, Jung Y. Radiation-induced liver disease: current understanding and future perspectives. Exp Mol Med 2017;49:e359.

36. Seppala J, Lahtinen T, Kolmonen P. Major reduction of monitor units with the avoidance of leaf-sequencing step by direct aperture based IMRT optimisation. Acta Oncol 2009;48:426-30.

37. Abo-Madyan Y, Aziz MH, Aly MM, et al. Second cancer risk after 3D-CRT, IMRT and VMAT for breast cancer. Radiother Oncol 2014;110:471-6.

38. Smith W, Menon G, Wolfe N, Ploquin N, Trotter T, Pudney D. IMRT for the breast: a comparison of tangential planning techniques. Phys Med Biol 2010;55:1231-41.

39. Mayo CS, Urie MM, Fitzgerald TJ. Hybrid IMRT plans: concurrently treating conventional and IMRT beams for improved breast irradiation and reduced planning time. Int J Radiat Oncol Biol Phys 
2005;61:922-32.

40. Xie $X$, Ouyang $S$, Wang $H$, et al. Dosimetric comparison of left-sided whole breast irradiation with 3D-CRT, IP-IMRT and hybrid IMRT. Oncol Rep 2014;31:2195-205. 